Supporting Information for

\title{
Probing Kinetics and Mechanism of Formation of Mixed Metallic Nanoparticles in a Polymer Membrane by Galvanic Replacement between two Immiscible Metals: Case Study of Nickel/Silver Nanoparticle Synthesis
}

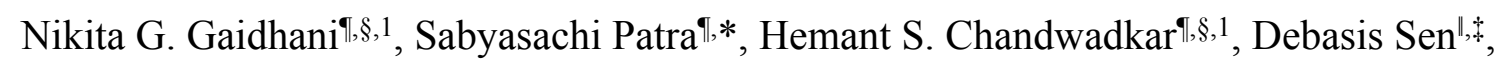
Chiranjib Majumdert,*, Shobha V. Ramagiri ${ }^{\ddagger}$ Jayesh R. Bellare ${ }^{\ddagger}$

『Radiochemistry Division, Bhabha Atomic Research Centre, Trombay, Mumbai 400085, India

$\S$ Department of Chemistry, Sandip University, Nashik, Maharashtra 422213, India

"Solid State Physics Division, Bhabha Atomic Research Centre, Mumbai 400085, India

+Chemistry Division, Bhabha Atomic Research Centre, Trombay, Mumbai 400085, India

¥Homi Bhabha National Institute, Anushaktinagar, Mumbai 400094, India

${ }^{\ddagger}$ Department of Chemical Engineering, IIT Bombay, Powai, Mumbai 400076, India

*Corresponding Author: Email: sspatra86@gmail.com, spatra@,barc.gov.in (S. Patra), Tel: +91-

22-2559-2469

${ }^{1}$ Formerly, Masters project student at Radiochemistry Division, BARC from Sandip University, Nashik, Maharashtra, India

Number of pages: S1 - S13

Number of figures: 7

Number of tables: 2 
Characterizations

Transmission electron microscopy (TEM) images of the membrane after

galvanic replacement reaction for various time period

SAXS analysis

Theoretical modeling of SAXS data

Fitted SAXS profiles

S7-S8

Synchrotron SAXS data

Diameter of silver nanoparticles as obtained using TEM and SAXS

Others

Calculation of number of AgNPs in the membrane

Determination of critical free energy barrier of nucleation

S11

Parameters of linear fit and the obtained rate constants ( $k$ ) of nucleation at the three different temperatures. 
Transmission electron microscopy (TEM) images of the membrane after galvanic replacement reaction for various time period
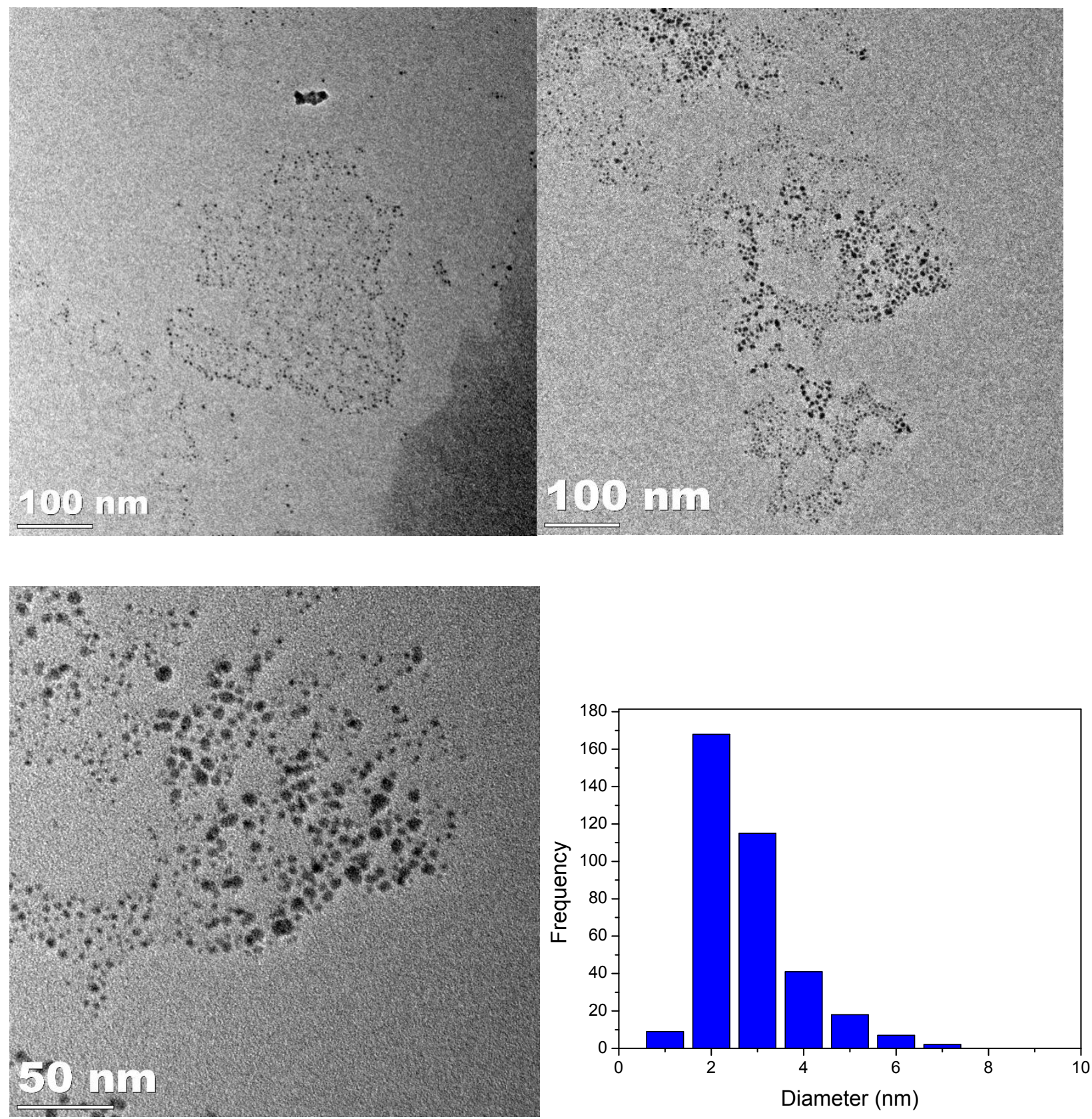

Figure S1. TEM images across the membrane thickness showing the evolution of nanoparticles in the bulk (interior) of the Nafion-117 membrane after galvanic replacement for 5 min and 
nanoparticle size distribution histogram. The histogram has been constructed considering 360 particles.

(a)

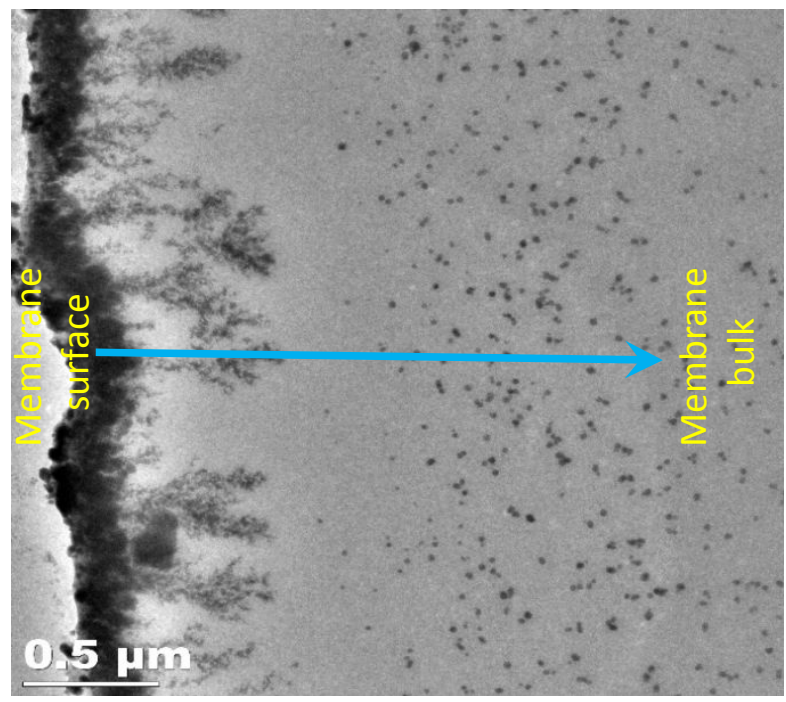

(b)
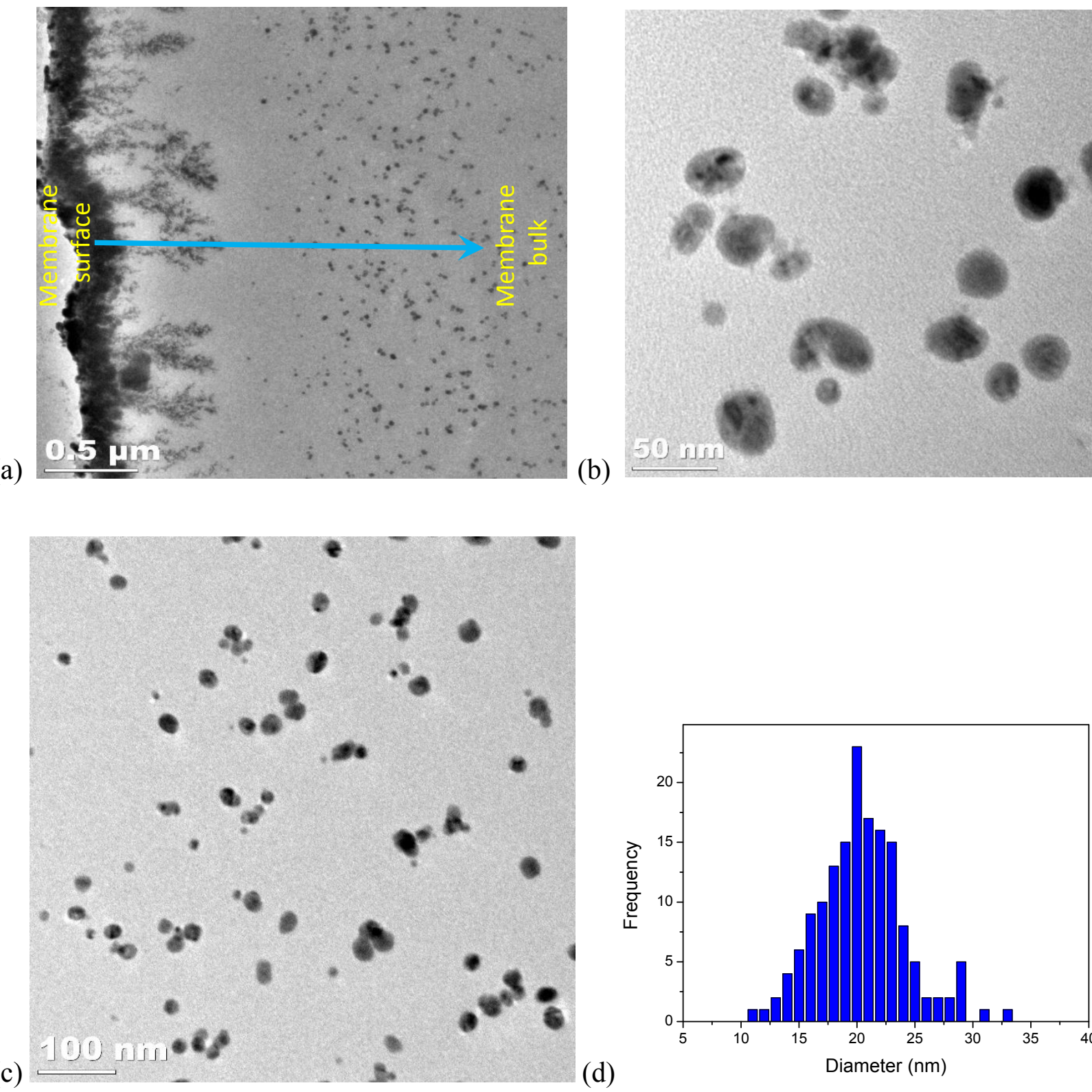

(d)

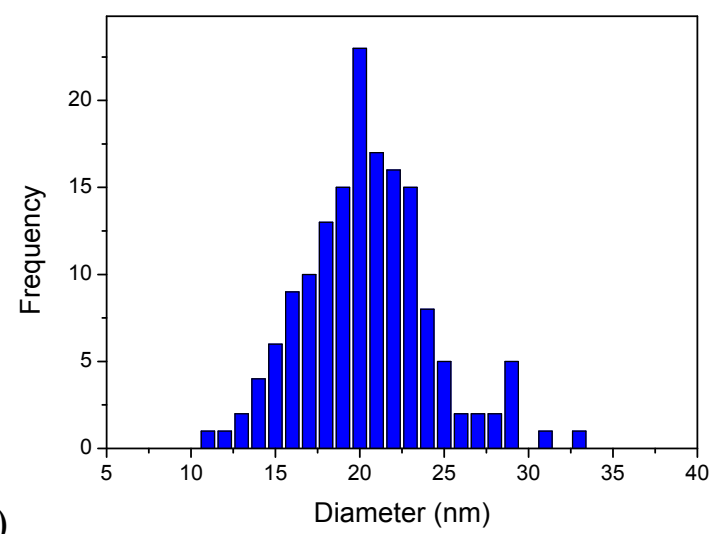

Figure S2. TEM images across the membrane thickness showing the evolution of nanoparticles near the surface (a) and in the bulk (b,c) of the Nafion-117 membrane after galvanic replacement for 20 min and the nanoparticle size distribution histogram (d). The blue arrow is eye escort 
showing the directions from surface to the bulk of the membrane. The histogram has been prepared considering total 158 nanoparticles present in bulk of the membrane only.

(a)
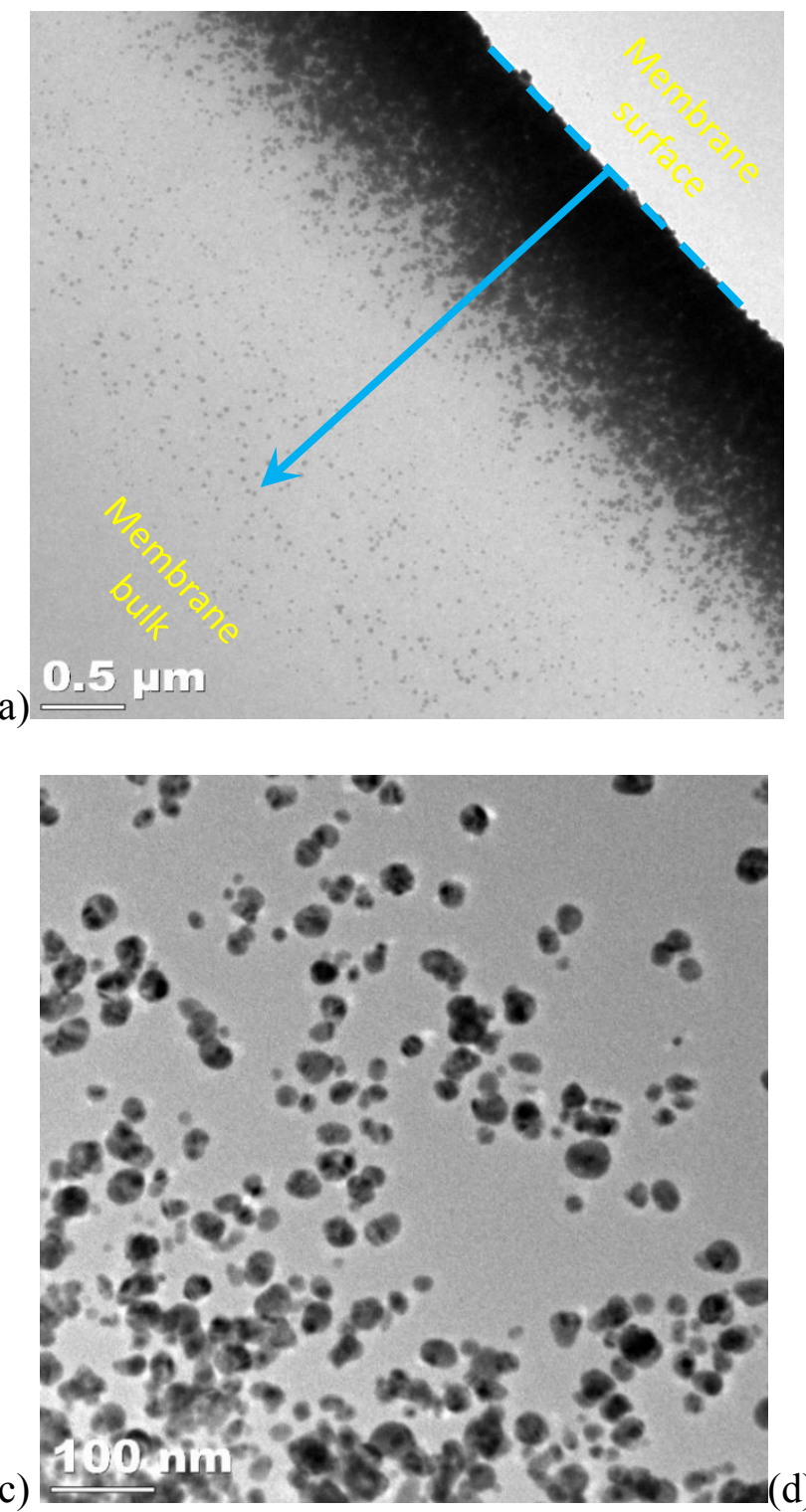

(b)
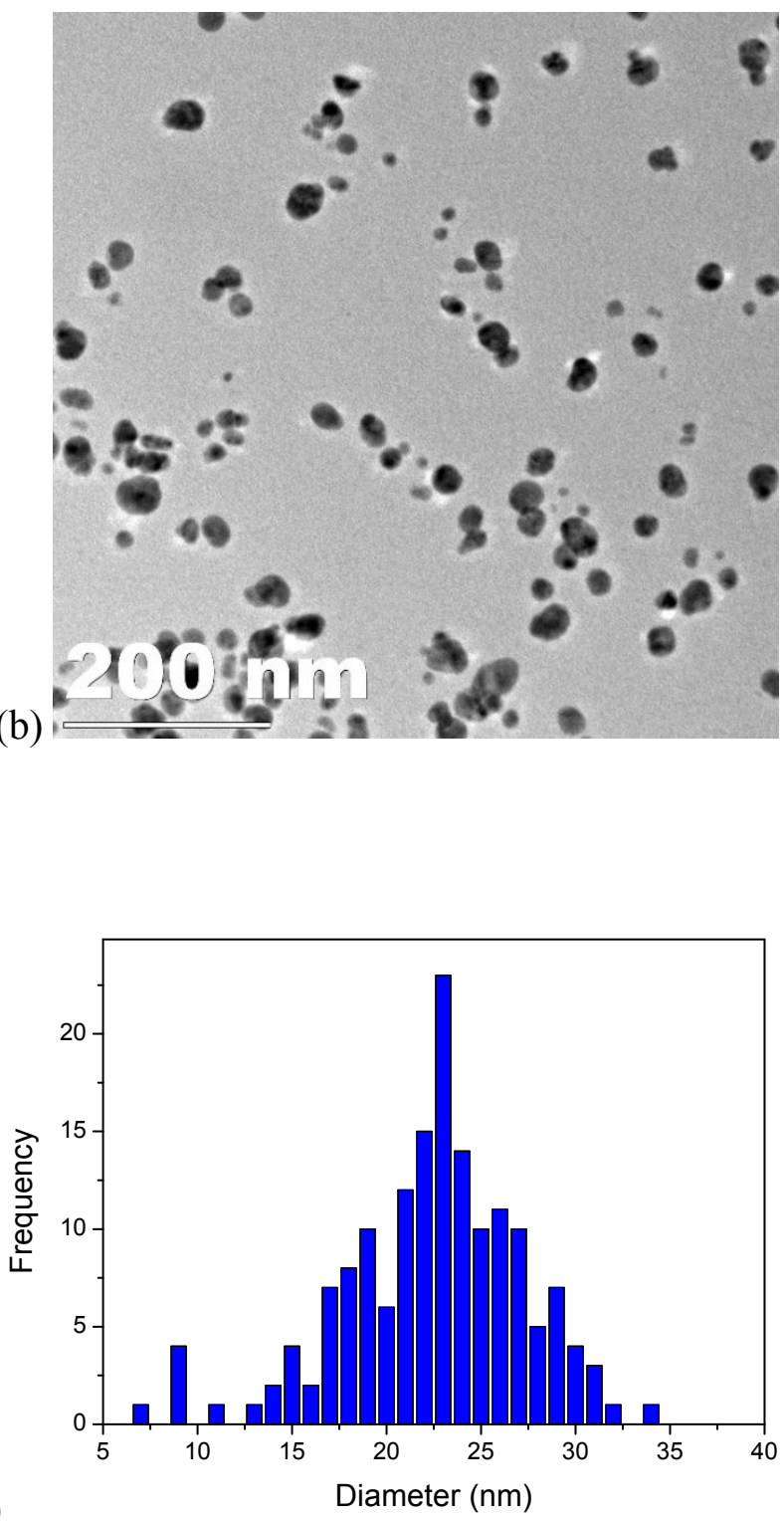

Figure S3. TEM images across the membrane thickness showing the evolution of nanoparticles near the surface (a) and in the bulk (b,c) of the Nafion-117 membrane after galvanic replacement for $60 \mathrm{~min}$ and the nanoparticle size distribution histogram (d). The blue arrow is eye escort showing the directions from surface to the bulk of the membrane. The histogram has been prepared considering total 162 nanoparticles present in bulk of the membrane only. 


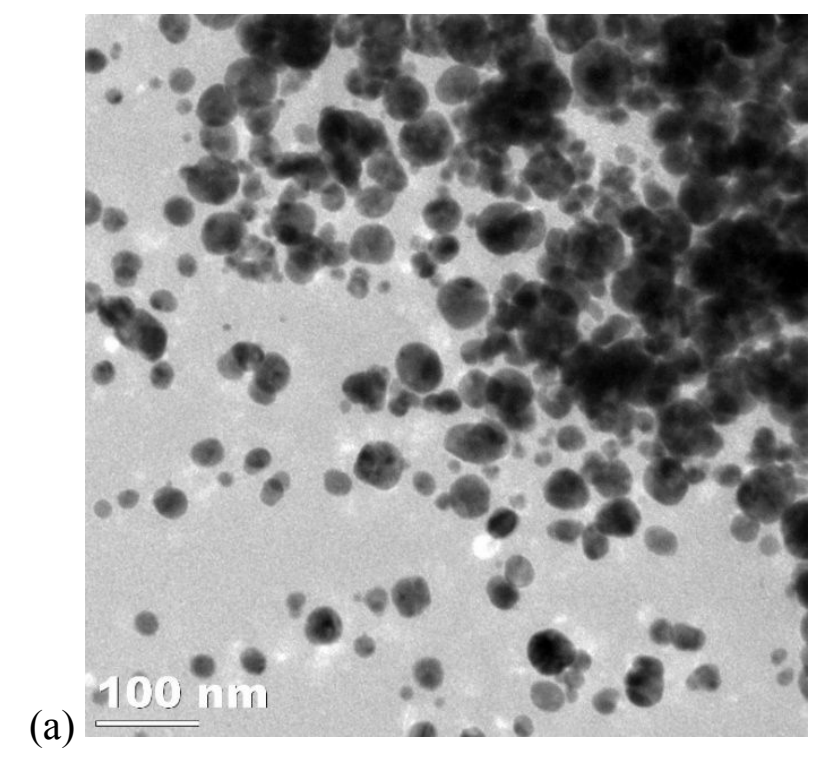

(b)
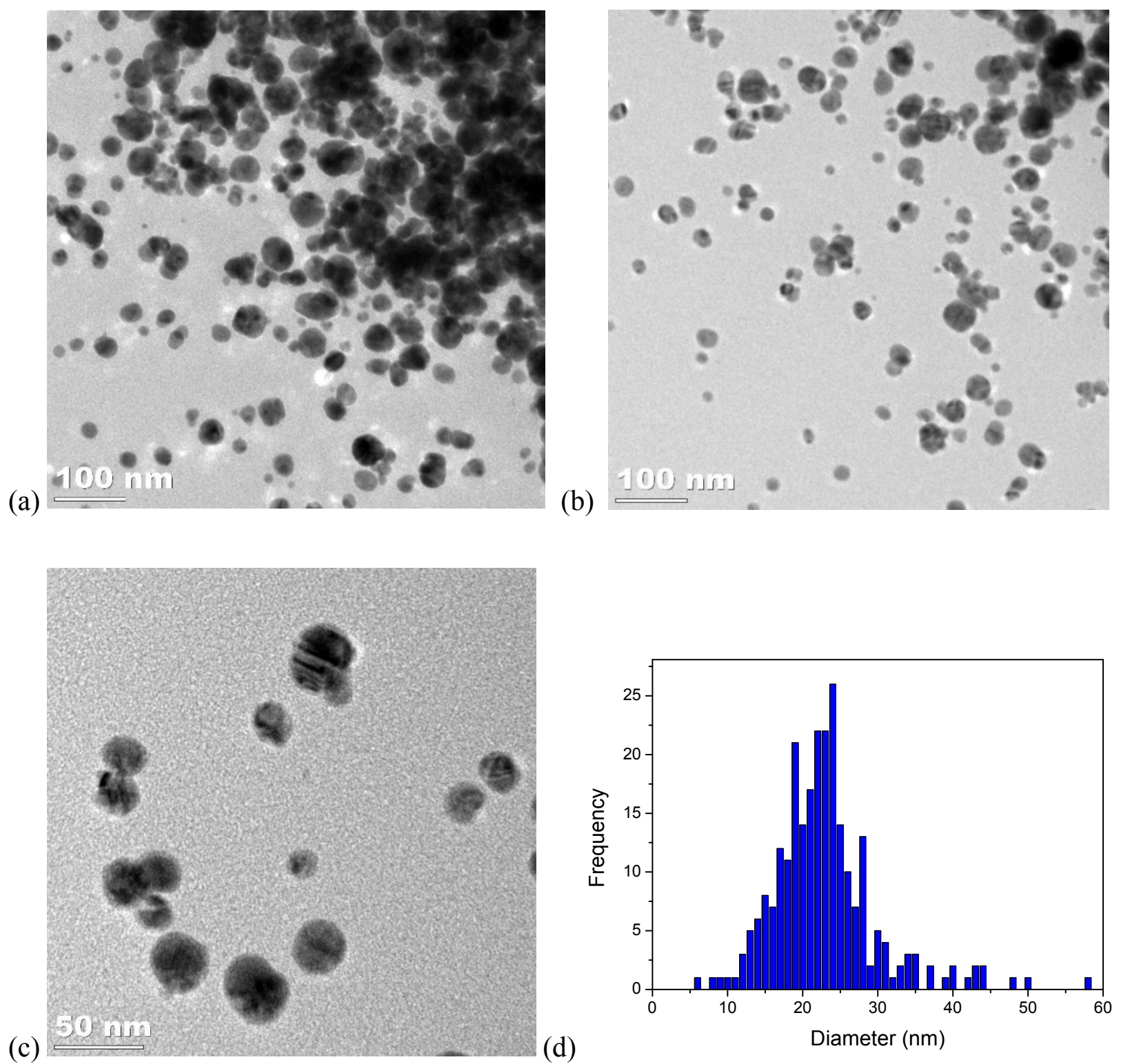

Figure S4. TEM images across the membrane thickness showing the evolution of nanoparticles near the surface (a) and in the bulk (b,c) of the Nafion-117 membrane after galvanic replacement for $120 \mathrm{~min}$ and the nanoparticle size distribution histogram (d). The blue arrow is eye escort showing the directions from surface layer to the bulk of the membrane. The histogram has been prepared considering 256 nanoparticles present in bulk of the membrane only. 


\section{SAXS analysis}

Nafion-117 membrane is very well characterized by small-angle X-ray scattering (SAXS) in literature. $^{1}$ In our earlier studies ${ }^{2-6}$ we have shown that SAXS is an excellent technique for morphological characterization of metal nanoparticles embedded in Nafion-117 membrane. The SAXS analysis begins with fitting of radial averaged SAXS profile of a pristine Nafion-117 membrane with a realistic and plausible model. Taking guidance from available literature on pristine Nafion-117 membrane, the pristine membrane SAXS profile was divided into two scattering vector $(\mathrm{q})$ regimes for fitting purpose. The scattering contribution at low $\mathrm{q}$ regime $(<1$ $\mathrm{nm}^{-1}$ ) originates from the membrane matrix (MM) and the scattering contribution at high q regime $\left(>1 \mathrm{~nm}^{-1}\right)$ originates from the reverse micellar water clusters (WC). The total scattering intensity for a pristine membrane was represented as the cumulative contributions from membrane matrix and water cluster:

$$
I(q)=C_{M M} I_{M M}(q)+C_{W C} I_{W C}(q)
$$

where $\mathrm{C}_{M M}$ and $\mathrm{C}_{W C}$ represents the scale factors and the subscripts $\mathrm{MM}$ and $\mathrm{WC}$ denotes membrane matrix and water clusters respectively. Polydisperse spherical particle model with mass fractal structure factor $(\mathrm{S}(\mathrm{q}, \mathrm{R}))^{7}$ was adopted to fit the $\mathrm{MM}$ contribution (Equation 2). Under local monodisperse approximation $^{8}$

$I_{M M}(q)=\int_{0}^{\infty} P(q, R) R^{6} D(R) S(q, R) d R$

Where, $\mathrm{P}(\mathrm{q}, \mathrm{R})$ is the form factor for sphere of radius $\mathrm{R}$ and can be expressed as Equation 3 .

$$
P(q, R)=9 \frac{(\sin (q R)-q R \cos (q R))^{2}}{(q R)^{6}}
$$


Where $\mathrm{D}(\mathrm{R})$ denotes the size distribution (considered as Lognormal) of the spherical object of radius $R$ and can be expressed as Equation 4.

$$
D(R)=\frac{1}{\sqrt{2 \pi \sigma^{2} R^{2}}} e^{-\frac{\ln \left(R / R_{0}\right)^{2}}{2 \sigma^{2}}}
$$

Where $R_{0}$ and $\sigma$ symbolizes the median radius and polydispersity index $(0<\sigma<1)$ of the distribution respectively.

The scattering contribution from the water clusters (WC) at high q regime was also analysed considering polydisperse spherical particle model. The term $\left(\mathrm{I}_{\mathrm{WC}}(\mathrm{q})\right)$ in Equation 1 under local monodisperse approximation can be stated as:

$$
I_{W C}(q)=\int_{0}^{\infty} P_{W C}(q, R) R^{6} D_{W C}(R) S_{W C}(q, R) d R
$$

where, $\mathrm{P}_{\mathrm{WC}}(\mathrm{q}, \mathrm{R})$ signifies the form factor for spherical water clusters and $\mathrm{D}_{\mathrm{WC}}(\mathrm{R})$ signifies the size distribution. $\mathrm{S}_{\mathrm{WC}}(\mathrm{q}, \mathrm{R})$ designates the structure factor (hard sphere) due to the inter water cluster interaction with hard sphere type interaction potential ${ }^{8}$ which causes the appearance of peak like feature at $\mathrm{q} \approx 1.7 \mathrm{~nm}^{-1}$.

The SAXS profiles of Nafion-117 membrane incorporated with metal nanoparticles consist of scattering contribution due to the membrane (MM and WC) and nanoparticles (NP). In order to fit the radial averaged SAXS profile of membrane incorporated with NiNPs, the total scattering intensity I(q) was projected as collective contribution of scattering from both membrane and NiNPs (Equation 6).

$$
I(q)=C_{M M} I_{M M}(q)+C_{W C} I_{W C}(q)+C_{N i N P} I_{N i N P}(q)
$$


Where $\mathrm{C}_{\mathrm{MM}}, \mathrm{C}_{\mathrm{WC}}$ and $\mathrm{C}_{\mathrm{NP}}$ are scale factors and the subscripts $M M, W C$ and NiNP represent membrane matrix, water cluster and nickel nanoparticle respectively. For all the three contributions, polydisperse spherical particle model (Equation 2) was used to fit the SAXS data. A hard sphere structure factor was also considered for NiNPs.

For the NiNPs loaded membrane samples subjected for galvanic replacement with $\mathrm{AgNO}_{3}$ solution, the scattering contribution from AgNPs was also included in Equation 6. The scattering contrast of the two metals was defined. The cumulative scattering intensity for such a membrane was approximated by Equation 7.

$I(q)=C_{M M} I_{M M}(q)+C_{W C} I_{W C}(q)+C_{N i N P} I_{N i N P}(q)+C_{A g N P} I_{A g N P}(q)$

where, the subscript $A g N P$ denotes to silver nanoparticles in the membrane. It is to be noted that, the parameters for the MM and WC kept constant in each case to keep the number of unknown parameters minimum.

\section{Fitted SAXS profiles}
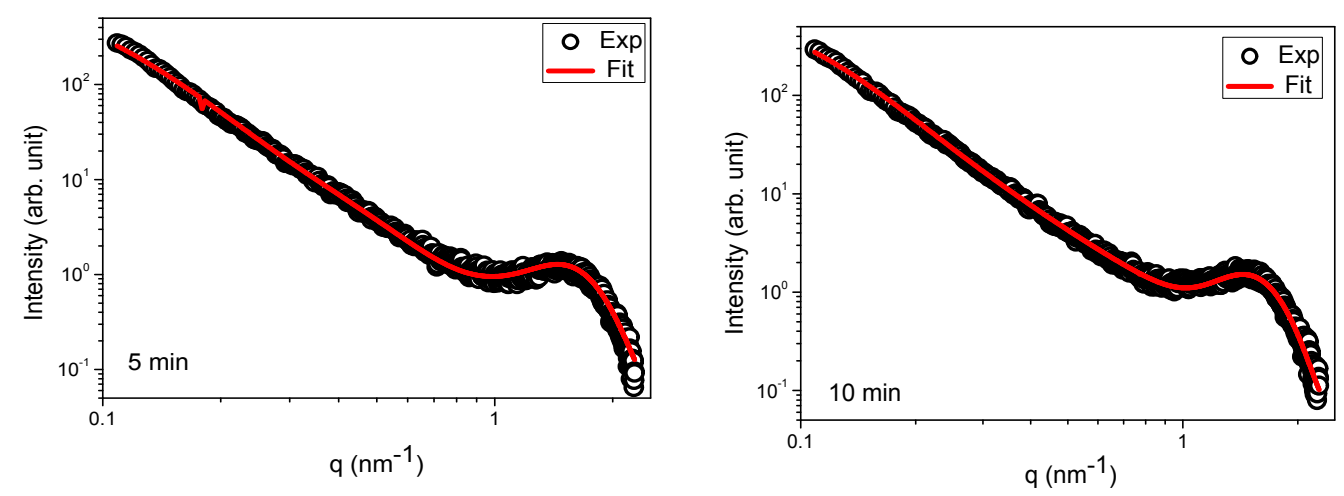

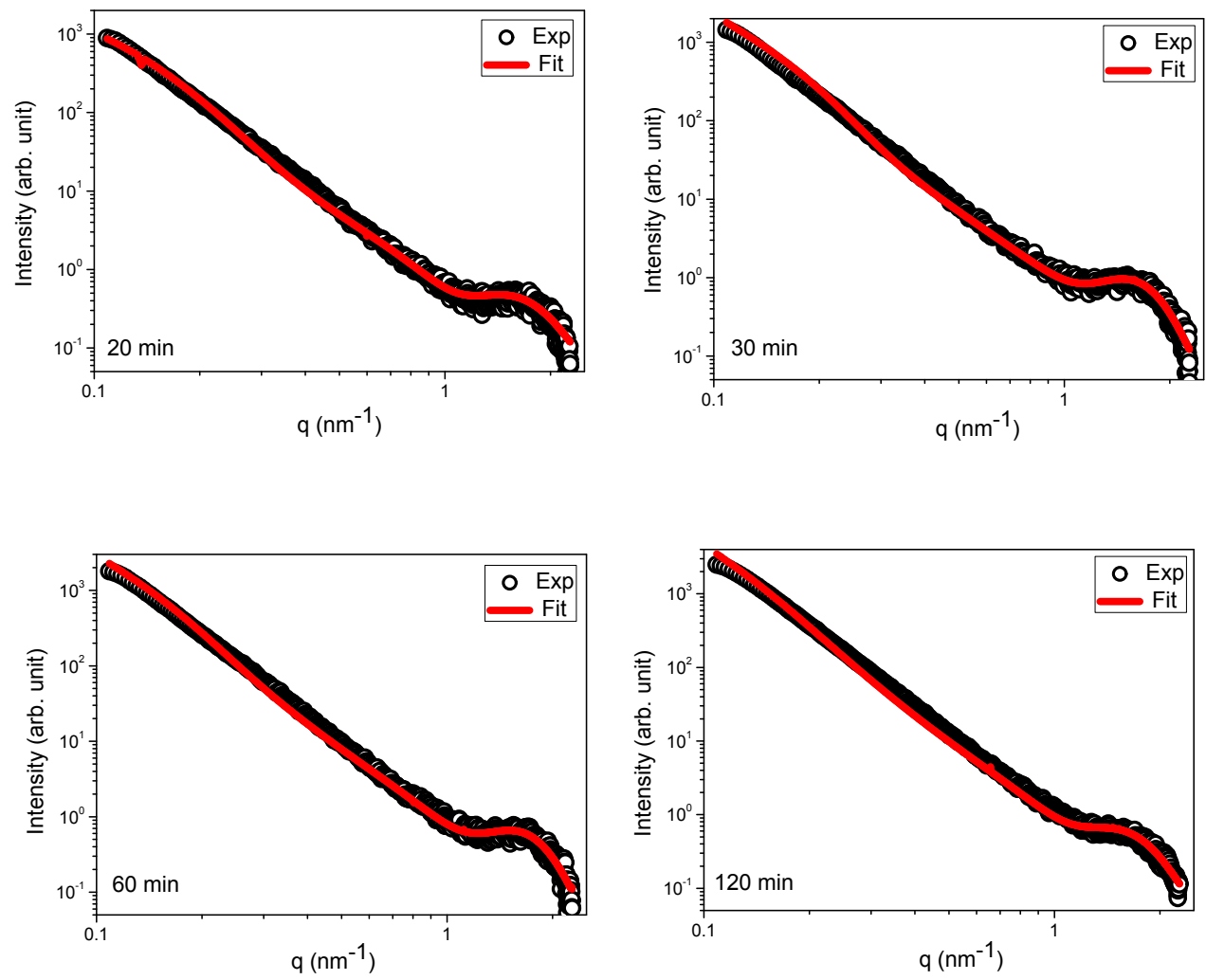

Figure S5. Fitted SAXS curve (laboratory experiments) at different reaction time. 

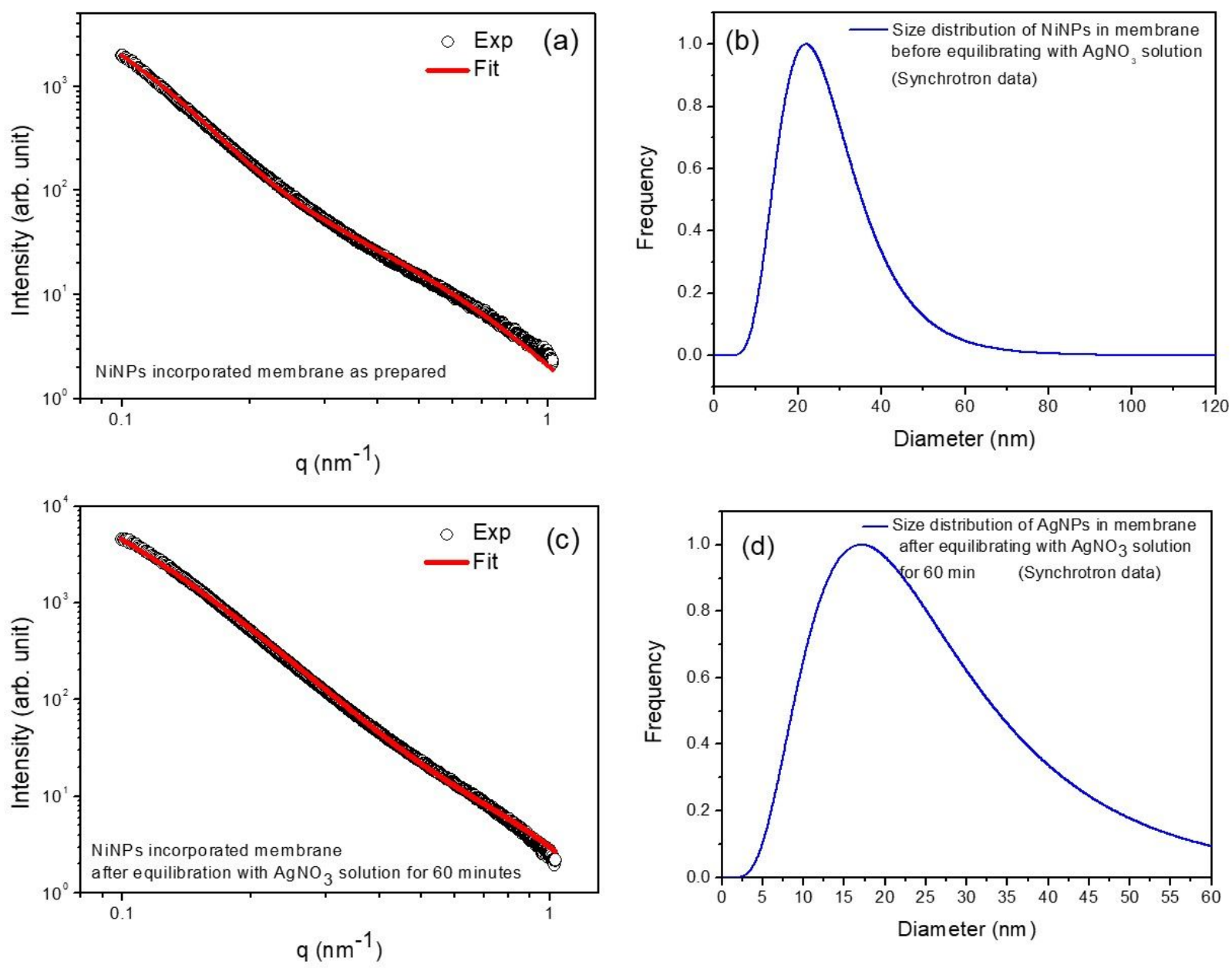

Figure S6. Fitted SAXS curve (synchrotron experiment at INDUS) and the respective size distribution histogram of the nanoparticles at $0 \mathrm{~min}(\mathrm{a}, \mathrm{b})$ and $60 \mathrm{~min}(\mathrm{c}, \mathrm{d})$ of galvanic replacement reaction respectively. 
Table S1. Diameter of silver nanoparticles as obtained using TEM and SAXS.

\begin{tabular}{|c|c|c|}
\hline \multirow{2}{*}{$\begin{array}{c}\text { Time for galvanic reaction } \\
(\mathrm{min})\end{array}$} & 2 & SAXS (median diameter) \\
\cline { 2 - 3 } & TEM (mode diameter) & 2 \\
\hline 5 & -- & 9 \\
\hline 10 & 20 & 18 \\
\hline 20 & -- & 22 \\
\hline 30 & 23 & 24 \\
\hline 60 & 24 & 24 \\
\hline 120 & & \\
\hline
\end{tabular}

\section{Calculation of number of AgNPs in the membrane.}

The results of SAXS analysis and radiotracer experiment have been combinedly used to calculate the number of AgNPs in the membrane of known volume using the following equation:

$$
N_{A g N P S}=\frac{\left(n_{A g} 0 \times 3 V_{m}\right)}{4 \pi r^{3}}
$$

Where $n_{A g^{0}}$ denotes the number of moles of $\mathrm{Ag}^{0}$ atoms present in the piece of membrane as AgNP at a given time as obtained from radiotracer study, $V_{m}$ denotes the molar volume of silver (10.28 $\left.\mathrm{cm}^{3} / \mathrm{mol}\right) ; \mathrm{r}$ denotes the median radius of the nanoparticles as obtained from SAXS data. 


\section{Determination of critical free energy barrier of nucleation.}

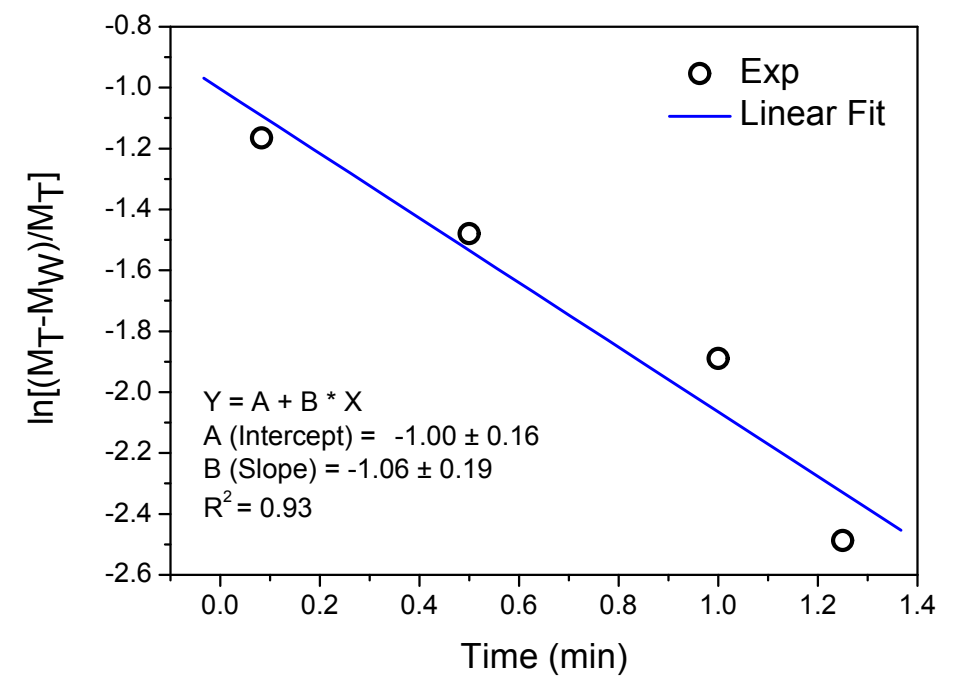

Figure S7. Plot of natural logarithm of mole fraction of silver monomer in the membrane with time during nucleation $\left(\mathrm{T}=30^{\circ} \mathrm{C}\right)$. The slope of the straight-line is correlated to the rate of nucleation at that temperature.

Table S2. Parameters of linear fit and the obtained rate constants $(\mathrm{k})$ of nucleation at the three different temperatures.

\begin{tabular}{|c|c|c|c|}
\hline $\begin{array}{c}\text { Temperature } \\
\left({ }^{\circ} \mathrm{C}\right)\end{array}$ & Slope & Intercept & $\mathrm{k}\left(\mathrm{s}^{-1}\right)$ \\
\hline 30 & -1.06 & -1.00 & 1.06 \\
\hline 45 & -21.21 & 5.23 & 21.2 \\
\hline 60 & -37.51 & 5.28 & 37.5 \\
\hline
\end{tabular}




\section{References}

1. Mauritz, K.A.; Moore, R.B. State of Understanding of Nafion. Chem. Rev. 2004, 104, $4535-4585$.

2. Patra, S.; Pandey, A.K.; Sen, D.; Ramagiri, S.V.; Bellare, J.R.; Mazumder, S.; Goswami, A. Redox Decomposition of Silver Citrate Complex in Nanoscale Confinement: An Unusual Mechanism of Formation and Growth of Silver Nanoparticles, Langmuir, 2014, 30, 2460-2469.

3. Patra, S.; Sen, D.; Pandey, A.K.; Agarwal, C.; Ramagiri, S.V.; Bellare, J.R.; Mazumder, S.; Goswami, A. Local Conditions Influencing In Situ Formation of Different Shaped Silver Nanostructures and Subsequent Reorganizations in Ionomer Membrane, J. Phys. Chem. C, 2013, $117,12026-12037$.

4. Naik, A.N.; Patra, S.; Kanekar, A.S.; Sen, D.; Ramagiri, S.V.; Bellare, J.R.; Mazumder, S.; Goswami, A. Nafion membrane incorporated with silver nanoparticles as optical test strip for dissolved hydrogen peroxide: Preparation, deployment and the mechanism of action, Sens. Actuators, B 2018, 255, 605-615.

5. Naik, A.N.; Patra, S.; Sen, D.; Goswami, A. Evaluating the mechanism of nucleation and growth of silver nanoparticles in a polymer membrane under continuous precursor supply: tuning of multiple to single nucleation pathway, Phys. Chem. Chem. Phys., 2019, 21, 4193-4199.

6. Patra, S.; Sen, D.; Pandey, A.K.; Bahadur, J.; Mazumder, S.; Ramagiri, S.V.; Bellare, J.R.; Roth, S.V.; Santoro, G.; Yu, S.; Goswami, A. Time resolved growth of membrane stabilized silver NPs and their catalytic activity, $R S C A d v ., 2014,4,59379$ - 59386. 
7. Teixeira, J. Small-angle scattering by fractal systems, J. Appl. Crystallography 1988, 21, 781785.

8. Pedersen, J.S. Determination of Size Distributions from Small-Angle Scattering Data for Systems with Effective Hard-Sphere Interactions. J. Appl. Cryst. Sci. 1994, 7, 595-608. 